Editorial

\title{
Megaloblastic anemia with pancytopenia in infancy:a rare entity
}

\author{
Prijo Philip ${ }^{1}$, Doddabasava K², Dhrithiman Shetty ${ }^{3}$
}

${ }^{1}$ Dr. Prijo Philip, Assistant Professor, Department of Pediatrics, K.S. Hegde Medical Academy, Mangalore, Karnataka, India, ${ }^{2}$ Dr. Doddabasava K, Chief Pediatrician, Taluk General Hospital, Sindhanur, Raichur, Karnataka, India, ${ }^{3}$ Dr. Dhrithiman Shetty, Assistant Professor, Department of Pediatrics, K.S. Hegde Medical Academy, Mangalore, Karnataka, India.

Corresponding Author: Dr. Prijo Philip, Department of Pediatrics, K.S. Hegde Medical Academy, Deralakatte, Mangalore, Karnataka, India. E-mail-prijophilipkk@gmail.com

\begin{abstract}
Megaloblastic anemia in infants present with generalized weakness, failure to thrive, or irritability. Diagnosis is usually centered on complete blood count and peripheral smear, which may show macrocytes, hyper segmented neutrophils, reticulocytopenia and a raised mean corpuscular volume $(\mathrm{MCV}>100 \mathrm{fl}$ ). Pancytopenia has also been noted. We report an exclusively breast fed six months old female child who presented with irritability, poor feeding and developmental delay. Her initial blood picture revealed pancytopenia, with normal MCV. Vitamin B12 levels were found to be reduced. Maternal levels of Vitamin B12 were also found to be borderline low. The child was treated as per protocols and improvement was evidenced with return of hematological parameters to normal and gradual advancement of milestones. The authors wish to underscore the importance ofMegaloblastic anemia as an important and rare cause of anemia in infancy. Early recognition and treatment are imperative to prevent catastrophic sequelae.
\end{abstract}

Keywords- Infants, Megaloblastic anemia, Pancytopenia

\section{Introduction}

Vitamin B12 is an important component of body metabolism. Nutritional deficiency of the same may seem trivial and is a known causative factor, but in developing countries, where maternal deficiency of Vitamin B12 invariably spells deficiency in the

\section{Case Presentation}

We present the case of a 6 months 20-day old female child, who presented to the Pediatric Outpatient Department with complaints of irritability, reduced feeding and multiple episodes of vomiting per day for 3 months prior to the presentation. Clinical examination revealed pallor and hepatomegaly.

Developmental assessment revealed that the child had Global Developmental Delay. Anthropometric parameters (weight, length and head circumference) were below third centile for age. Laboratory examination revealed Hemoglobin-5.5g/dL, White Blood Cell count-3500/mm3, Platelet-87000/mm3, MCV-97 fL(normal level 72-88 fL), MCH-32.2 pg (normal level 24-30 pg), Reticulocyte count-1.2\% and LDH-2825 U/L. The peripheral blood examination reported that red blood cells were macrocytic and neutrophils were hyper segmented with pancytopenia (Fig. 1,2).

Bone marrow aspiration revealed hypercellular marrow with $\mathrm{M}: \mathrm{E}$ ratio of $0.5: 1$, erythroid hyperplasia with megalonormoblastic reaction (Fig. 3). Serum Vitamin B12 level was found to be $65 \mathrm{pg} / \mathrm{mL}$ (normal level 211$911 \mathrm{pg} / \mathrm{mL}$ ). The maternal Serum VitaminB12 level was found to be $213.7 \mathrm{pg} / \mathrm{mL}$ (normal level 211-911pg/ml).

Manuscript received: $28^{\text {th }}$ December 2017

Reviewed: $7^{\text {th }}$ January 2018

Author Corrected: $14^{\text {th }}$ January 2018

Accepted for Publication: $18^{\text {th }}$ January 2018 
Editorial
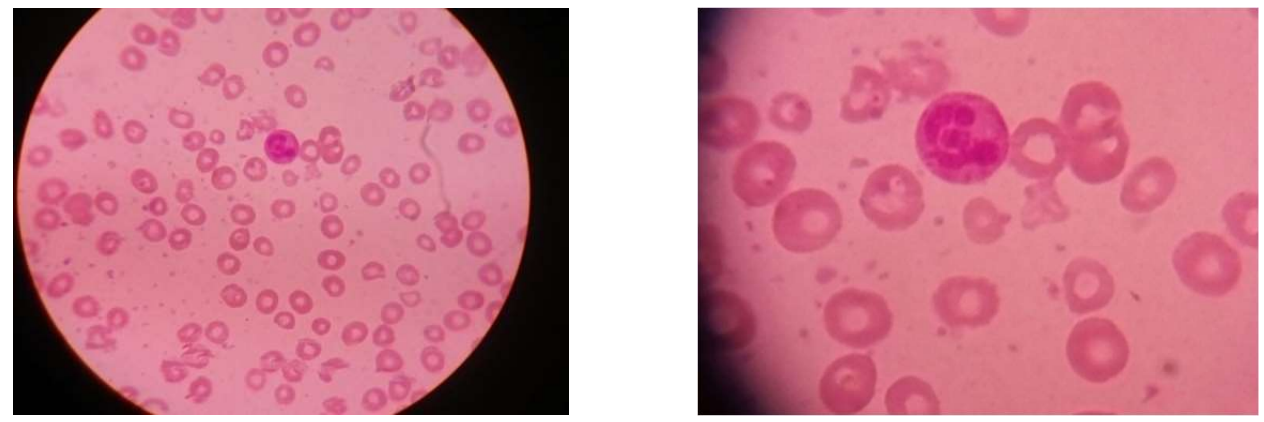

Fig-1: Depiction of microcytosis and pancytopenia Fig-2: Hypersegmented neutrophils in blood film

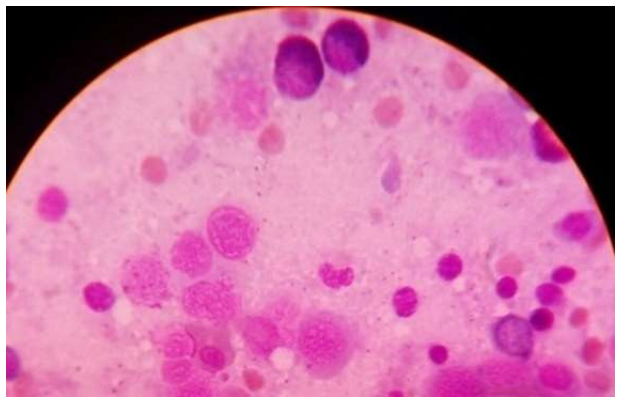

Fig-3: Hypercellular marrow and erythroid hyperplasia

The child was treated with $250 \mathrm{mcg}$ Vitamin B12 intramuscularly once a week for 4 weeks followed by oral Vitamin B12 supplementation. Following treatment, hemoglobin levels and other relevant hematological parameters rapidly improved, preceding a gradual clinical improvement. Further follow up revealed improvement in all domains of development, although milestones were still delayed, compared for age.

\section{Discussion}

Vitamin B12 (Cobalamin) has been attributed to play a huge role in human intermediary metabolism. It is paramount, for the conversion of methyl-malonyl-CoA to succinyl-CoA (a compound metabolized by the Krebs cycle to produce energy) and also to ensure the optimal activity of an enzyme called methionine synthase, that catalyzes the methylation of homocysteine, culminating in the formation of the essential amino acid methionine [1]. Vitamin B12 deficiency, therefore, leads to the accumulation of methylmalonic acid and homocysteine, and these heralds the beginning of various clinical, hematological, neurological and psychiatric manifestations in the child [2].

Cobalamin also functions as a cofactor for methylmalonyl CoA mutase, the enzyme that plays a major role in the conversion of methylmalonyl CoA to succinyl CoA, which is a prerequisite for the metabolism of various odd-chain fatty acids and also for purine and pyrimidine synthesis. Methylmalonic aciduria and defective amino acid synthesis results from the lack of this essential cofactor, manifesting as pancytopenia, metabolic acidosis as well as hypotonia [3]. Various factors such as inborn errors of metabolism and nutritional problems can result in deficient levels of Vitamin B12 in infancy. The most frequent inborn error, that has been described, is cobalamin $\mathrm{C}$ disorder, which has been attributed to a mutation of the $M M A C H C$ gene [4]. Nutritional causes are however more common and most infants found to have B12 deficiency have been noted to be born to mothers with low vitamin B12 levels and who have been exclusively breastfed.

It follows then, that the breast milk of vegan mothers is frequently poor in vitamin B12, and their newborn infants run the possibility of having have low vitamin stores [1]. Rarer causes of deficiency include the surgical removal of the stomach and /or distal ileum, autoimmune gastritis, Crohn disease, exocrine pancreatic insufficiency and Whipple disease[5].

Vitamin B12 deficiency has now been recognized as an important cause of infant morbidity and mortality throughout the world, and this is more so in the Indian subcontinent, Mexico, Central America and certain regions of Africa [6]. Infantile Vitamin B12 deficiency is important to recognize because early recognition and 


\section{Editorial}

treatment can prevent potentially fatal neurological sequelae [7]. A positive co-relation has been found between maternal and breastfed infant vitamin $\mathrm{B}_{12}$ deficiency linked to deficient B12 levels in breast milk. Specker and colleagues did postulate that vitamin B12 milk concentrations less than $360 \mathrm{pmol} / \mathrm{L}$, approximately corresponding to a maternal serum $\mathrm{B} 12$ of less than $300 \mathrm{pmol} / \mathrm{L}$, could potentially result in vitamin B12 deficiency in infancy [5]. It has been stated previously that the offspring of women with low vitamin B12 levels during the period of pregnancy and lactation may have reduced stores of the vitamin at birth [8]. Such children run the risk of developing signs of vitamin B12 deficiency during infancy, generally not prior to the fourth month of life, although neonatal cases have been reported[9].

Childhood vitamin B12 deficiency appears to be more significant than previously thought, and has been considered a rarity, especially in infancy [10]. It has been stated previously that approximately one-third of low-income women and children in Guatemala have deficient $(<148 \mathrm{pmol} / \mathrm{L})$ or marginal $(148-220 \mathrm{pmol} / \mathrm{L})$ levels of plasma vitamin B12, and that the lowest levels bear a stark relation to abject growth and development [11]. In the Indian population, individuals tend to be partial to a vegetarian diet; therein vitamin B12 deficiency during pregnancy is common[12]. It has been reported that the infants of such mothers seem to be affected by a syndrome characterized by mild developmental regression and alterations in skin pigmentation[13].

A significant question at this juncture would be the apparent lack of observable signs and symptoms among deficient pregnant mothers. This may be attributable to the fact that due to the consumption of a diet inclusive of large amounts of vegetables that contain high folate concentrations, the hematological effects of vitamin B12 deficiency may be masked. Moreover, the neuropsychiatric manifestations of vitamin B12 deficiency are usually mild in adolescents and adults, and stand a high chance of being overlooked[1]. On the contrary, breastfed infants born to vegan mothers develop substantial neurological damage. Importantly, these may persist and result in long-term cognitive and developmental delay irrespective of adequate therapy and the complete disappearance of hematological problems[14]. The clinical features of infantile vitamin B12 deficiency depends on the severity of the deficient state. Many children may be asymptomatic, some may present with megaloblastic anemia, diagnosed only via hematological profile[15]. Symptomatic infants usually present between 2 and 12 months of age, when neonatal stores of vitamin B12 have been depleted and dietary sources prove inadequate. Typical symptoms include poor feeding, weight loss and irritability. Other reported features include glossitis and susceptibility to infections [16]. The neurological manifestations are profound and include irritability, apathy, lethargy and regression of the gross motor developmental milestones. Routine plotting of the head circumference on follow up visits will allow the noting of receding values of the same, indicative of regression of brain growth.

Gradually, children progress to hypotonia with hyperreflexia, and also exhibit choreo-athetoid movements. Demye-lination, delayed myelination, impaired methylation and lactate accumulation in the peripheral nerves, spinal cord and cerebrum have all been hypo-thesized as being responsible for these profound neurological mani-festations, although no clear putative factor has been recognized[17].

The diagnosis of vitamin $\mathrm{B}_{12}$ deficiency in infants may be confirmed by determining the serum vitamin $\mathrm{B}_{12}$ concentration, although it is not an absolute requirement for initiating treatment. Bone marrow studies may also confirm the diagnosis [5]. Treatment regimens for Vitamin B12 deficiency include both intramuscular and oral replacement, although oral supplementation in infants is fraught with inherent issues such as regurgitation and vomiting. The British Columbia Guidelines and Protocols Advisory Committee have recommended oral $\mathrm{B}_{12}$ replacement therapy of $1000 \mu$ gdaily [5].

As was observed in our patient, treated vitamin B12 and iron deficient infants tend to improve dramatically, the hematological values returning quickly to the normal state, and the neurological signs thereby progressively decrease[1]. That being said, the severe cases described thus far have also identified permanent sequelae[14]. In our case, it was also imperative to address the issue of borderline low maternal levels of Vitamin B12, that required extensive dietary planning.

\section{Conclusion}

The authors wish to use this case to highlight the varied signs, symptoms and complications of Vitamin B12 deficiency in infancy, as well as the need to consider vitamin B12 deficiency in infants presenting with severe anemia even if their hematological parameters may not indicate megaloblastic anemia, attributable to the concomitant presence of iron deficiency that may modify the presentation. 


\section{Editorial}

What this study adds to existing knowledgeMegaloblastic anemia is a well-researched entity. However, megaloblastic anemia in infantile period attributable to deficiency in maternal nutrition is a rare entity and surplus literature is not available for the same. The authors wish to highlight the consequences of said deficiency and enlighten readers with respect to current guidelines for treatment, that may aid in halting progress and improve prognostication.

Funding: Nil, Conflict of interest: None initiated, Perission from IRB: Yes

\section{References}

1. Guez S, Chiarelli G, Menni F, Salera S, Principi N, Esposito S. Severevitamin B12 deficiency in an exclusively breast fed 5-month-old Italian infantborn to a mother receiving multivitamin supplementation during pregnancy. BMC Pediatr. 2012 Jun 24;12:85. doi: 10.1186/1471-2431-12-85.

2. Stabler SP, Allen RH, Savage DG, Lindenbaum J. Clinical spectrum and diagnosis of cobalamin deficiency. Blood.1990Sep1;76(5):871-81.

3. Kapadia CR. Vitamin B12 in health and disease: part I--inherited disorders of function, absorption, and transport. Gastroenterologist. 1995 Dec;3(4):329-44.

4. Watkins D, Rosenblatt DS. Inborn errors of cobalamin absorption and metabolism. Am J Med Genet C Semin Med Genet. 2011 Feb 15; $157 \mathrm{C}$ (1):3344. doi: 10.1002/ajmg.c.30288. Epub 2011 Feb 10.

5. Roumeliotis N, Dix D, Lipson A. Vitamin B(12) deficiency in infants secondary to maternal causes. CMAJ. 2012 Oct 2;184(14):1593-8. doi: 10.1503/cmaj. 112170. Epub 2012 Jun 18.

6. Stabler SP, Allen RH. Vitamin B12 deficiency as a worldwide problem. Annu Rev Nutr. 2004;24:299-326.

7. Graham SM, Arvela OM, Wise GA. Long-term neurologic consequences of nutritional vitamin B12 deficiency in infants. J Pediatr. 1992 Nov;121(5 Pt 1): $710-4$.

8. Baker H, Frank O, Deangelis B, Feingold S, Kaminetzky HA. Role of placenta in maternal- fetalvitamintransfer in humans. Am J Obstet Gynecol. 1981 Dec1;141(7):792-6.

9. Erdeve O, Arsan S, Atasay B, Ileri T, Uysal Z. A breast-fed newborn with megaloblastic anemia-treated with the vitamin B12 supplementation of the mother. J Pediatr Hematol Oncol. 2009 Oct;31(10):763-5.

10. Kömür M, Bayram İ, Erbey F, Küçükosmanoğlu O. A Rare Cause of Thrombocytopenia in Infants: Vitamin B12 Deficiency. Eur J Gen Med [Internet]. 2010 [cited 2016 Sep 12]; 7(1). Available from: http://dergipark. ulakbim. gov.tr/ejgm/article/view/5000114928

11. Jones KM, Ramirez-Zea M, Zuleta C, Allen LH. Prevalent vitamin B-12 deficiency in twelve-month-old Guatemalan infants is predicted by maternal B-12 deficiency and infant diet. J Nutr. 2007May;137 5): 307-13.

12. Katre P, Bhat D, Lubree H, Otiv S, Joshi S, Joglekar C, Rush E, Yajnik C. Vitamin B12 and folic acid supplementation and plasmatotal homocysteine concentrations in pregnantIndianwomen with lowB12 and high folatestatus. Asia Pac J Clin Nutr. 2010; 19(3): 335-43.

13. JADHAV M, WEBB JK, VAISHNAVA S, BAKER SJ. Vitamin B12 deficiency in Indian infants. A clinical syndrome. Lancet. 1962 Nov 3;2(7262):903-7.

14. Honzik T, Adamovicova M, Smolka V, Magner M, Hruba E, Zeman J. Clinical presentation and metabolic consequences in 40 breast fedinfants with nutritional vitamin B12 deficiency--what have we learned? Eur J Paediatr Neurol.2010Nov;14(6):488-95. doi: 10.1016/j. ejpn.2009.12.003. Epub2010 Jan 20.

15. Wong MP, Wadsworth L, Wu JK, Dix D. Case 2: A pale infant - not a typical case of iron deficiency. Paediatr Child Health. 2008 Jul;13(6):507-11.

16. Kaikov Y, Wadsworth LD, Hall CA, Rogers PC. Transcobalamin II deficiency: case report and review of the literature. Eur J Pediatr. 1991 Oct;150(12):841-3.

17. Dror DK, Allen LH.Effectof vitamin B12 deficiency on neurodevelopment in infants: current knowledge and possiblemechanisms.Nutr Rev.2008 May; 66 (5): 250-5. doi: 10.1111/j.1753-4887.2008.00031.x.

\section{How to cite this article?}

Prijo Philip, Doddabasava K, Dhrithiman Shetty. Megaloblastic anemia with pancytopenia in infancy:a rare entity. Int J Pediatr Res. 2018;5(1):3-6.doi:10.17511/ijpr.2018.i01.02. 